\title{
A new fast finite element method for dislocations based on interior discontinuities
}

\author{
Robert Gracie $^{1}$, Giulio Ventura ${ }^{2}$ and Ted Belytschko ${ }^{1, *, \dagger}$ \\ ${ }^{1}$ Department of Mechanical Engineering, Northwestern University, 2145 Sheridan Road, Evanston, \\ IL 60208-3111, U.S.A. \\ ${ }^{2}$ Department of Structural Engineering and Geotechnics, Politecnico di Torino, 10129 Torino, Italy
}

\begin{abstract}
SUMMARY
A new technique for the modelling of multiple dislocations based on introducing interior discontinuities is presented. In contrast to existing methods, the superposition of infinite domain solutions is avoided; interior discontinuities are specified on the dislocation slip surfaces and the resulting boundary value problem is solved by a finite element method. The accuracy of the proposed method is verified and its efficiency for multi-dislocation problems is illustrated. Bounded core energies are incorporated into the method through regularization of the discontinuities at their edges. Though the method is applied to edge dislocations here, its extension to other types of dislocations is straightforward. Copyright (c) 2006 John Wiley \& Sons, Ltd.
\end{abstract}

Received 25 August 2006; Accepted 30 August 2006

KEY WORDS: dislocations; extended finite element method

\section{INTRODUCTION}

The study of fundamental phenomena in plasticity often involves the simulation of the motion of thousands of dislocations; however, the cost of these computations is often very large and the limiting factor on model size. So the development of computationally more efficient dislocation models is desirable. This paper presents a new numerical technique based on the extended finite element method (XFEM) of Belytschko and Black [1], and Moës et al. [2]. In this method,

\footnotetext{
*Correspondence to: Ted Belytschko, Department of Mechanical Engineering, Northwestern University, 2145 Sheridan Road, Evanston, IL 60208-3111, U.S.A.

†E-mail: tedbelytschko@northwestern.edu

Contract/grant sponsor: Office of Naval Research; contract/grant number: N00014-03-1-1097

Contract/grant sponsor: Army Research Office; contract/grant number: W911NF-05-1-0049
} 
using the tangential enrichment of Belytschko et al. [3], dislocations are modelled directly as interior discontinuities in the standard finite element method. The cost of a computation is roughly comparable to that of a standard finite element model of the same size. The method is applied to edge dislocations here; however, its extension to other types of dislocations is straightforward.

Roughly speaking, dislocations models can be categorized as either continuum based, such as the method of van der Giessen and Needleman [4], or discrete, such as the methods of Amodeo and Ghoniem [5], Schwarz and Tersoff [6], Kubin and co-workers [7], and Zbib et al. [8].

In the continuum-based approach, the solution of a domain with $n_{d}$ dislocations is determined by superimposing the analytical infinite domain solution of each dislocation and an image stress field. The solution is obtained by a finite element model (FEM) with boundary tractions chosen to cancel the tractions of the infinite dislocation fields. A disadvantage of these methods is that at each quadrature point on the boundary of the FE domain, a sum over all $n_{d}$ dislocations must be performed. When a dislocation core is near a boundary, the accurate integration of the tractions requires a large number of quadrature points. Furthermore, calculation of the driving stress at each of the $n_{d}$ dislocations required the superposition of $n_{d}$ infinite domain fields, which introduces an $n_{d}^{2}$ dependence in the computational cost. So, the cost of the superposition calculations becomes quite large for many dislocations. Moreover, the standard FEM cannot capture a discontinuity in the displacement field within a single element; the slip across the glide plane is only captured in an average sense.

In discrete dislocation models, a dislocation is modelled by a string of line segments. The PeachKoehler force on each line segment is computed by the superposition of the analytical infinite domain solution of each of the $n_{s}$ dislocation segments plus the image stresses. In these models, the image stresses are generally computed approximately from empirical/analytical formulae, which are generally valid only for simple boundary geometries. According to Devincre et al. [9], one of the greatest difficulties in such models is that the boundary condition are not met exactly.

In the discrete-continuum method of Lemarchand et al. [10], plastic strains from a discrete dislocation model are imposed at the quadrature points of a FE model through a homogenization. Such an approach smears the slip due to dislocations onto the FE mesh and avoids the use of superposition. Conceptually this approach is similar to the one proposed here, but by means of the XFEM we can precisely model the discontinuities.

A common characteristic of most dislocation methods is the use of superposition and analytical infinite-domain solutions for a dislocation. This leads to the need to determine correction stresses, i.e. image stresses, and contributes to large computational costs. A method is therefore desirable which does not depend on analytical solutions, does not require the computation of image stresses nor uses superposition.

XFEM allows arbitrary discontinuities to be modelled within a finite element mesh; it was first applied to two-dimensional crack propagation in an elastic domain without remeshing $[1,2]$. The method was extended to three dimensions by Sukumar et al. [11] and to nonlinear fracture mechanics by Moës and Belytschko [12]. XFEM has been applied to shear bands by Song et al. [13], inclusions and holes by Sukumar et al. [14], and two-phase flow by Chessa and Belytschko [15]. Some other recent applications are [16-22]; of particular similarity are applications to shear bands [23, 24].

In this paper, a dislocation is modelled in a manner closely akin to the dislocation model of Volterra [25] and Eshelby [26]. In the Volterra model, a cut is introduced into an elastic solid, as shown in Figure 1(a). The surfaces of the cut are displaced, the material is then reattached, as in Figure 1(b), and the stresses are determined. In the proposed method a cut is introduced along the 


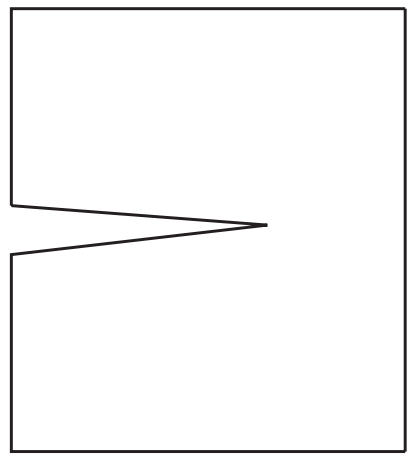

(a)

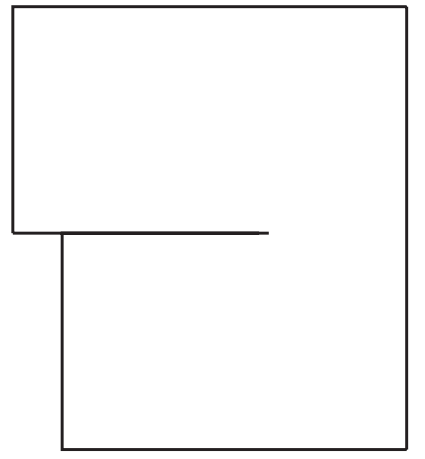

(b)

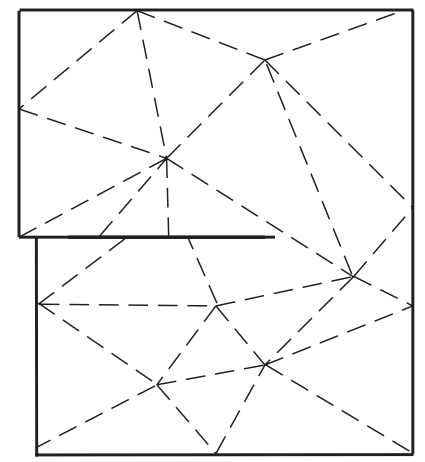

(c)

Figure 1. Illustration of the construction of a Volterra dislocation.

slip line and the top surface is moved relative to the bottom by the dislocation strength. The two sides of the discontinuity are then reconnected and the stress field is determined by the governing equations of the solid. This of course could be done with a standard finite element method if the element edges coincide with the slip line, but it would be very difficult if not impossible for arbitrary directions of slip lines and many dislocations. The ability of XFEM to model arbitrary discontinuities is its principal benefit to the present work. With XFEM the slip across the glide plane can be modelled exactly within a single element.

Recently, Ventura et al. [27] applied the partition of unity method (PUM) of Melenk and Babuška [28], to edge dislocations. In this method, the solution for an edge dislocation in an infinite domain was obtained by an enrichment by the partition of unity. Slip along the glide plane was imposed by constraining the enriched degrees of freedom. The practicality of this method for large numbers of dislocations was limited by the need to integrate the singular infinite domain solution; the partition of unity in fact was the weak link, since computation of dislocation stress field enrichments is very expensive. In the proposed method we introduce non-singular enrichment functions which lead to a method which is suitable for the modelling of large numbers of dislocations.

This paper is organized as follows. In the next section the governing equations are given; in Section 3 enrichment functions for edge dislocations are introduced and the discrete equations are derived; in Section 4 the proposed method is verified and examples with multiple dislocations are presented; conclusions are presented in Section 5.

\section{GOVERNING EQUATIONS}

Consider the domain $\Omega$ bounded by $\Gamma$ shown in Figure 2 with tractions $\overline{\mathbf{t}}$ defined on boundary $\Gamma_{t}$, displacements $\overline{\mathbf{u}}$ defined on boundary $\Gamma_{u}$ and with internal discontinuities $\Gamma_{d}^{\alpha}$, each of which represents a dislocation $\alpha$. For convenience we define $\Gamma_{d}=\bigcup_{\alpha} \Gamma_{d}^{\alpha}$. Given the spaces

$$
\mathscr{U}_{0}=\left\{\mathbf{u} \in C^{0}, \mathbf{u}=0 \text { on } \Gamma_{u}, \mathbf{u} \text { is discontinuous on } \Gamma_{d}\right\}
$$




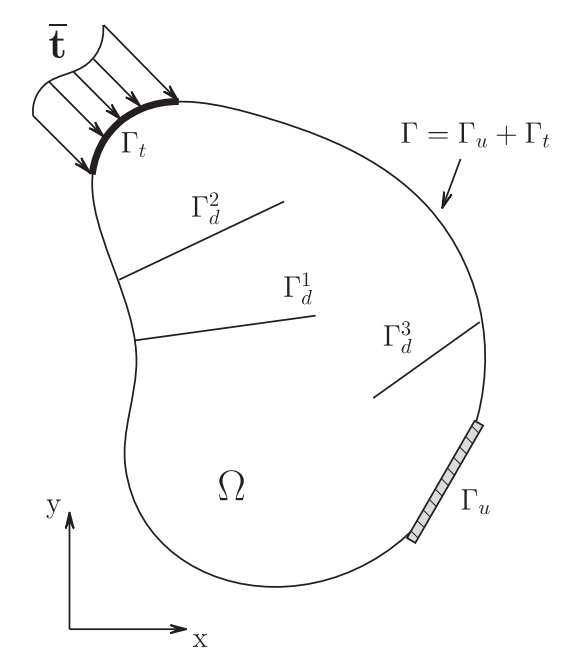

Figure 2. Domain definition and notation.

and

$$
\mathscr{U}=\left\{\mathbf{u} \in C^{0}, u=\overline{\mathbf{u}} \text { on } \Gamma_{u}, \mathbf{u} \text { is discontinuous on } \Gamma_{d}\right\}
$$

we solve the weak form of the equilibrium equation: find $\mathbf{u} \in \mathscr{U}, \ni$

$$
\int_{\Omega} \boldsymbol{\varepsilon}(\mathbf{v})^{\mathrm{T}}: \boldsymbol{\sigma}(\boldsymbol{\varepsilon}(\mathbf{u})) \mathrm{d} \Omega-\int_{\Omega} \mathbf{g} \cdot \mathbf{v} \mathrm{d} \Omega-\int_{\Gamma_{t}} \overline{\mathbf{t}} \cdot \mathbf{v} \mathrm{d} \Gamma=0, \quad \forall \mathbf{v} \in \mathscr{U}_{0}
$$

where $\boldsymbol{\sigma}$ is the Cauchy stress, $\mathbf{g}$ is the body force per unit volume, and $\overline{\mathbf{t}}$ is the prescribed traction on the traction boundary $\Gamma_{t}$. A small strain, linear elastic formulation will be adopted. The strain displacement relation is

$$
\varepsilon_{i j}=\frac{1}{2}\left(u_{i, j}+u_{j, i}\right)
$$

and the constitutive model is

$$
\boldsymbol{\sigma}=\mathbf{C}: \varepsilon
$$

where $\mathbf{C}$ is the elasticity matrix.

\section{EDGE DISLOCATION ENRICHMENT FUNCTIONS}

We consider an edge dislocation, as illustrated in Figure 3. The geometry is described by the location of the dislocation core and the orientation of the glide plane. The glide plane of dislocation $\alpha$ is described by an affine function of the coordinates, $f^{\alpha}(\mathbf{x})=0$ where

$$
f^{\alpha}(\mathbf{x})=\alpha_{0}+\alpha_{j} x_{j}
$$



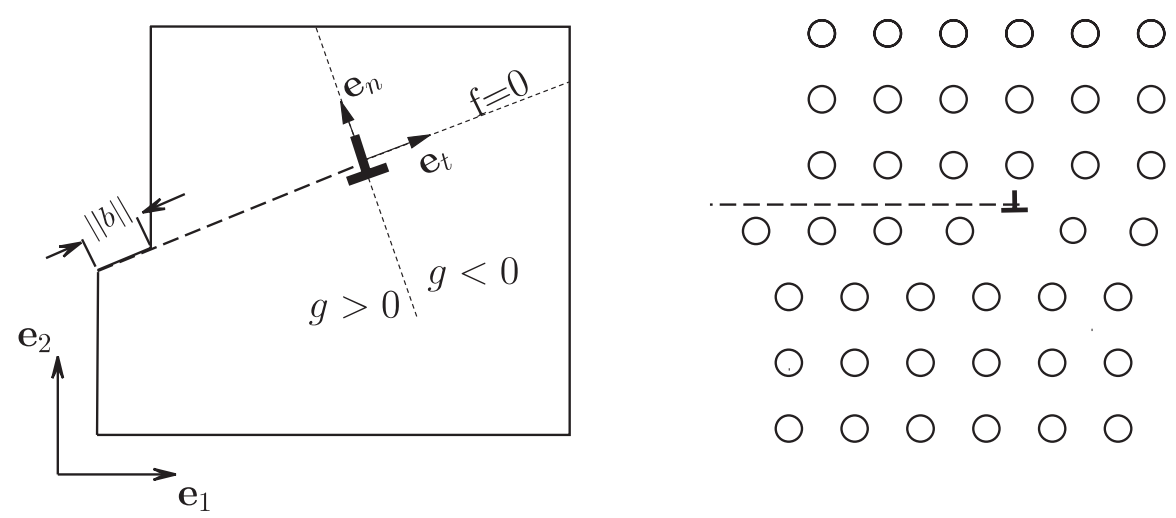

Figure 3. Description of an edge dislocation by functions $f(\mathbf{x})$ and $g(\mathbf{x})$. Dashed line represents the glide plane, $\mathbf{b}$ is Burgers vector.

and repeated indices denote summations. The active slip plane is described by the intersection of the glide plane with another plane $g^{\alpha}(\mathbf{x})$ where

$$
g^{\alpha}(\mathbf{x})=\beta_{i}+\beta_{j} x_{j}
$$

The coefficients of $g^{\alpha}(\mathbf{x})$ are normalized so that it is a distance function.

\subsection{Glide plane enrichment}

An edge dislocation $\alpha$ is characterized by a glide plane, a core and a Burgers vector, $\mathbf{b}^{\alpha}$. The glide plane is a strong discontinuity with a jump of $\mathbf{b}^{\alpha}$ in the displacement field; for an edge dislocation, $\mathbf{b}^{\alpha}$ is tangent to the glide plane. Slip along the glide plane is introduced by adding, as in the Volterra model, a prescribed internal discontinuity to the displacement field. For this purpose, the tangential step enrichment proposed by Belytschko et al. [3] will be used. The displacement approximation with tangential enrichment for an edge dislocation with Burgers vector $b^{\alpha} \mathbf{e}_{t}^{\alpha}$ has the following form:

$$
\mathbf{u}(\mathbf{x})=\sum_{I \in \mathscr{S}} N_{I}(\mathbf{x}) \mathbf{d}_{I}+\sum_{\alpha=1}^{n_{D}} b^{\alpha} \mathbf{e}_{t}^{\alpha} \sum_{J \in \mathscr{S}^{\alpha}} N_{J}(\mathbf{x}) H\left(f^{\alpha}(\mathbf{x})\right) \Psi\left(g^{\alpha}(\mathbf{x})\right)
$$

where $\mathscr{S}$ is the set of all nodes, $\mathscr{S}^{\alpha}$ is the set of enriched nodes to be defined later, $n_{D}$ is the number of dislocations, $N_{I}$ are the standard finite element shape functions, $\mathbf{d}_{I}$ are the nodal displacement degrees of freedom, $f^{\alpha}(\mathbf{x})$ is the function (6) defining glide plane $\alpha, g^{\alpha}(\mathbf{x})$ is the function (7) defining the location of core $\alpha, \mathbf{e}_{t}^{\alpha}$ is a unit tangent along glide plane $\alpha, H(z)$ is the symmetrized Heaviside function given by

$$
H(z)= \begin{cases}-1 / 2, & z<0 \\ 1 / 2, & z>0\end{cases}
$$

and $\Psi\left(g^{\alpha}(\mathbf{x})\right)$ will be discussed later. We call the second term in (8) an enrichment. The nodes that are enriched by the tangential step function, i.e. those in the set $\mathscr{S}^{\alpha}$, are shown in Figure 4. 


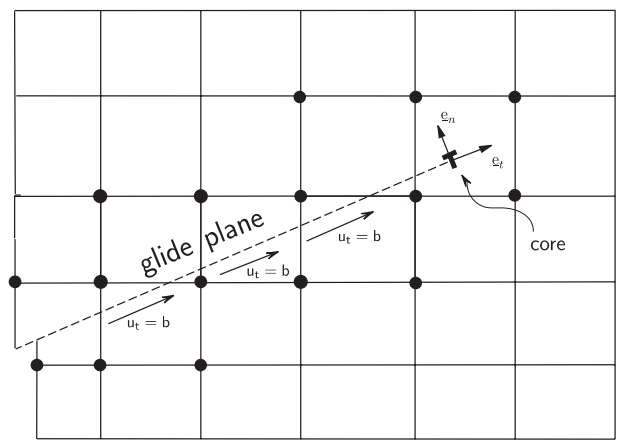

Figure 4. Illustration of the tangential enrichment scheme. Dashed line represents the glide plane and black circles represent nodes of the set $\mathscr{S}^{\alpha}$ that are enriched.

It can be seen that $\mathscr{S}^{\alpha}$ consists of all nodes of elements with an edge cut by glide plane $\alpha$. This type of enrichment was motivated by the PUM of Melenk and Babuška [28].

The displacement jump, [|u|], across glide plane $\alpha$ for $g^{\alpha}(\mathbf{x})>0$ is

$$
[|\mathbf{u}|]=\mathbf{u}^{+}(\overline{\mathbf{x}})-\mathbf{u}^{-}(\overline{\mathbf{x}})
$$

where $\mathbf{u}^{+}$and $\mathbf{u}^{-}$are the displacements above and below the glide plane, respectively. Inserting the displacement approximation (8) into (10) and simplifying we have

$$
\begin{aligned}
& {[|\mathbf{u}|]=\left[H^{+}-H^{-}\right] \Psi(\overline{\mathbf{x}}) b^{\alpha} \mathbf{e}_{t}^{\alpha} \sum_{J \in \mathscr{S}^{\alpha}} N_{J}(\overline{\mathbf{x}})} \\
& {[|\mathbf{u}|]=\Psi(\overline{\mathbf{x}}) b^{\alpha} \mathbf{e}_{t}^{\alpha} \sum_{J \in \mathscr{S}^{\alpha}} N_{J}(\overline{\mathbf{x}})}
\end{aligned}
$$

where $\overline{\mathbf{x}}$ solves $f^{\alpha}(\mathbf{x})=0$, and $H^{+}$and $H^{-}$are the values of the symmetrized Heaviside function (9) above and below the glide plane, respectively. Since $\sum_{J \in \mathscr{S}^{\alpha}} N_{J}(\overline{\mathbf{x}})=1$, where $\mathscr{S}^{\alpha}$ is the set of enriched nodes for dislocation $\alpha$, it follows from (11) that for any element that does not contain the core (i.e. where $\Psi(\mathbf{x})=1$ )

$$
[|\mathbf{u}|]=b^{\alpha} \overline{\mathbf{e}}_{t} \equiv \mathbf{b}^{\alpha}
$$

So the slip across the glide plane is equal to $b^{\alpha}$.

\subsection{Dislocation core enrichment}

The tangential displacement jump profile in analytical dislocation solutions is a step function, Figure 5(a). Such a profile is reproduced by approximation (8) with $\Psi(\mathbf{x})=H(g(\mathbf{x}))+1 / 2$. The strain energy when $\Psi(\mathbf{x})=H(g(\mathbf{x}))+1 / 2$ is unbounded and the displacement field is not admissible. We have developed two methods for regularizing $\Psi(\mathbf{x})$ and defining an admissible displacement field.

In the first regularization method we follow Eshelby [26] and introduce the tapered Somigliana dislocation shown in Figure 5(b). The tapered Somigliana dislocation has finite strain energy and 


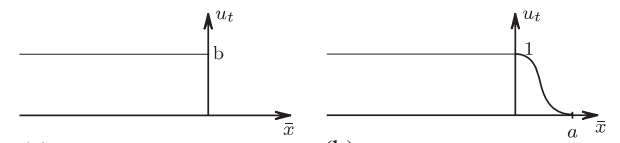

(a) (b)

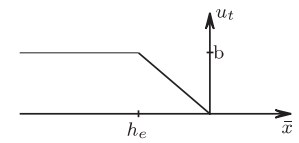

(c)

Figure 5. Illustration of different glide plane slip profiles. $u_{t}$ is the tangential jump across the glide plane, b is Burgers vectors, and $h_{e}$ is the mesh size: (a) is the tangential step discontinuity of the analytical solution; (b) is the tapered Somigliana dislocation; and (c) is the linear regularized dislocation.

is defined as

$$
\mathscr{P}(g(\mathbf{x}))= \begin{cases}1 & \text { if } g(\mathbf{x})>0 \\ \frac{\left(a^{m}-g(\mathbf{x})^{m}\right)^{n}}{a^{m n}} & \text { if }-a<g(\mathbf{x})<0 \\ 0 & \text { if } g(\mathbf{x})<-a\end{cases}
$$

where $n, m$ and $a$ are parameters that can be fit to atomistic/experimental results. The infinite energy dislocation core can be viewed as a special case of the tapered Somigliana dislocation, (13), where $a \rightarrow 0$.

The displacement approximation for the first regularization method is given by (8) with $\Psi(\mathbf{x})=\mathscr{P}(g(\mathbf{x}))$, so

$$
\mathbf{u}(\mathbf{x})=\sum_{I \in \mathscr{S}} N_{I}(\mathbf{x}) \mathbf{d}_{I}+\sum_{\alpha=1}^{n_{D}} b^{\alpha} \mathbf{e}_{t}^{\alpha} \sum_{J \in \mathscr{S}^{\alpha}} N_{J}(\mathbf{x}) H\left(f^{\alpha}(\mathbf{x})\right) \mathscr{P}\left(g^{\alpha}(\mathbf{x})\right)
$$

When high accuracy calculations are required, the introduction of such functions will allow the proposed method to incorporate a more realistic core behaviour.

When a quantitative measure of the strain energy is not necessary a second regularization method is to adopt a linear regularization such as shown in Figure 5(c). A triangular element is superimposed on the element containing the dislocation core, as shown in Figure 6. Two of the nodes of the superimposed element are taken as the nodes of the underlying core element edge which is cut by the glide plane. The third node $Q$ is selected so that the dislocation core will be located on the edge of the superimposed element. The enrichment for discontinuity $\alpha$ is taken as

$$
\mathbf{u}^{\alpha}(\mathbf{x})=b^{\alpha} \mathbf{e}_{t}^{\alpha} \sum_{J \in \overline{\mathcal{S}}^{\alpha}} \bar{N}_{J}(\mathbf{x}) H\left(f^{\alpha}(\mathbf{x})\right)
$$

where we have set $\Psi(\mathbf{x})=1$ in (8) and $\overline{\mathscr{S}}^{\alpha}$ is the set of nodes $\mathscr{S}^{\alpha}$ with the node $Q$ of the superimposed element excluded. The shape functions $\bar{N}_{J}$ are identical to those in (8) except that the shape functions of the superimposed element are used for the element which contains the dislocation core. This method was used in the examples presented here.

For a given mesh the strain energy from the linear regularization method is finite; however, since the regularization is mesh dependent, the strain energy diverges as the mesh is refined; this result is consistent with the fact that the regularization in Figure 5(c) tends to that in Figure 5(a), as the element size $h_{e} \rightarrow 0$. 


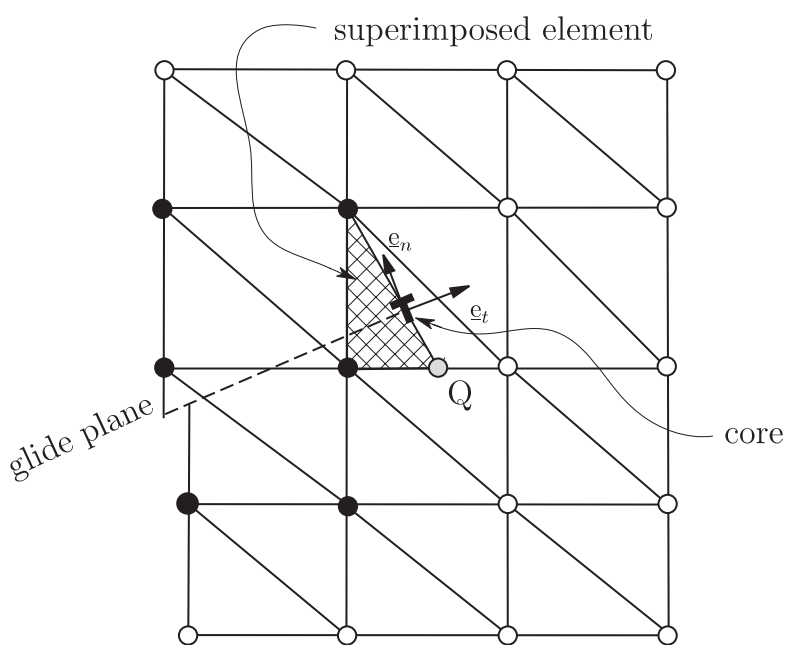

Figure 6. Illustration of the linear regularization of the step discontinuity. The cross-hatched triangle represents the superimposed element; the dashed line represents the glide plane; solid black nodes are the nodes in the set $\overline{\mathscr{S}}^{\alpha}$. The solid grey node is the unenriched node $Q$ of the superimposed element.

\subsection{Discrete equations}

Substituting the approximation (8) and an identical approximation for the test function $\mathbf{v}(\mathbf{x})$ into the weak form of the equilibrium (3), the following discrete equations are obtained

$$
\left[\begin{array}{ll}
\mathbf{K}_{d d} & \mathbf{K}_{d b} \\
\mathbf{K}_{d b}^{\mathrm{T}} & \mathbf{K}_{b b}
\end{array}\right]\left\{\begin{array}{l}
\mathbf{d} \\
\boldsymbol{\beta}
\end{array}\right\}=\left\{\begin{array}{c}
\mathbf{f}^{\mathrm{ext}} \\
\mathscr{B}
\end{array}\right\}
$$

where $\mathbf{d}=\left[\begin{array}{llll}\mathbf{d}_{1} & \mathbf{d}_{2} & \ldots & \mathbf{d}_{n}\end{array}\right]^{\mathrm{T}}$ are the standard nodal degrees of freedom and $n$ is the number of nodes. The vector $\boldsymbol{\beta}=\left[\begin{array}{llll}\mathbf{b}^{1} & \mathbf{b}^{2} \ldots \mathbf{b}^{n_{d}}\end{array}\right]^{\mathrm{T}}$ consists of the slips along the glide planes and the vector $\mathscr{B}=\left[\begin{array}{llll}\mathscr{B}^{1} & \mathscr{B}^{2} & \ldots & \mathscr{B}^{n_{d}}\end{array}\right]^{\mathrm{T}}$ is the reaction forces along the glide planes, where $n_{d}$ is the number of dislocations. The other terms are given by

$$
\begin{aligned}
\mathbf{K}_{I J}^{d d} & =\int_{\Omega / \Gamma_{d}} \mathbf{B}_{I}^{\mathbf{T}} \mathbf{C} \mathbf{B}_{J} \mathrm{~d} \Omega, \quad I \in \mathscr{S}, \quad J \in \mathscr{S} \\
\mathbf{K}_{I \alpha}^{d b} & =\int_{\Omega / \Gamma_{d}} \mathbf{B}_{I}^{\mathbf{T}} \mathbf{C D}_{\alpha} \mathrm{d} \Omega, \quad I \in \mathscr{S}, \quad \alpha=1,2, \ldots, n_{d} \\
\mathbf{K}_{\lambda \alpha}^{d b} & =\int_{\Omega / \Gamma_{d}} \mathbf{D}_{\lambda}^{\mathbf{T}} \mathbf{C D}_{\alpha} \mathrm{d} \Omega, \quad \lambda, \alpha=1,2, \ldots, n_{d} \\
\mathbf{f}^{\mathrm{ext}} & =\int_{\Omega} \mathbf{N}^{\mathbf{T}} \mathbf{g} \mathrm{d} \Omega+\int_{\Gamma_{t}} \mathbf{N}^{\mathbf{T}} \mathbf{t} \mathrm{d} \Gamma
\end{aligned}
$$


where

$$
\mathbf{B}_{I}=\left[\begin{array}{cc}
N_{I}(\mathbf{x})_{, x} & 0 \\
0 & N_{I}(\mathbf{x})_{, y} \\
N_{I}(\mathbf{x})_{, y} & N_{I}(\mathbf{x})_{, x}
\end{array}\right]
$$

and

$$
\mathbf{D}_{\alpha}=\sum_{I \in \mathscr{S}^{\alpha}}\left[\begin{array}{c}
\left(H\left(f^{\alpha}(\mathbf{x})\right) N_{I}(\mathbf{x}) \Psi(\mathbf{x})\right)_{, x} \mathbf{e}_{t}^{\alpha} \cdot \mathbf{e}_{x} \\
\left(H\left(f^{\alpha}(\mathbf{x})\right) N_{I}(\mathbf{x}) \Psi(\mathbf{x})\right)_{, y} \mathbf{e}_{t}^{\alpha} \cdot \mathbf{e}_{y} \\
\left(H\left(f^{\alpha}(\mathbf{x})\right) N_{I}(\mathbf{x}) \Psi(\mathbf{x})\right)_{, x} \mathbf{e}_{t}^{\alpha} \cdot \mathbf{e}_{y}+\left(H\left(f^{\alpha}(\mathbf{x})\right) N_{I}(\mathbf{x}) \Psi(\mathbf{x})\right)_{, y} \mathbf{e}_{t}^{\alpha} \cdot \mathbf{e}_{x}
\end{array}\right]
$$

Note that the definition of $\mathscr{S}^{\alpha}$ depends on the regularization used.

The Burgers vectors $\mathbf{b}^{\alpha}$ defining the slips are assumed to be given, so their effect appears in the discrete equations as an additional force. The nodal displacements are obtained by the first equation in (16) which gives

$$
\mathbf{d}=\mathbf{K}_{d d}^{-1}\left(\mathbf{f}^{\mathrm{ext}}-\mathbf{K}_{d b} \boldsymbol{\beta}\right)
$$

Note that $\mathbf{K}_{d d}$ is independent of the location, number and geometry of the dislocations and therefore does not change for a given mesh as the dislocations move or as new dislocations are nucleated. In a quasi-static analysis $\mathbf{K}_{d d}$ needs only to be inverted once for the entire simulation.

\subsection{Numerical implementation}

The form of the enrichment terms of displacement approximations (8) is not convenient for imposing displacement boundary conditions since $\mathbf{u}\left(\mathbf{x}_{I}\right) \neq \mathbf{d}_{I}$. To remedy this situation, we shift each enrichment function by a constant, as suggested by Belytschko et al. [3] and Ventura et al. [27]. The shifted displacement approximation is

$$
\mathbf{u}(\mathbf{x})=\sum_{I \in \mathscr{S}} N_{I}(\mathbf{x}) \mathbf{d}_{I}+\sum_{\alpha=1}^{n_{D}} \sum_{J \in \mathscr{S}^{\alpha}} N_{J}(\mathbf{x})\left[H\left(f^{\alpha}(\mathbf{x})\right) \Psi\left(g^{\alpha}(\mathbf{x})\right)-H_{J}^{\alpha} \Psi_{J}^{\alpha}\right] b^{\alpha} \mathbf{e}_{t}^{\alpha}
$$

where $H_{J}^{\alpha}=H\left(f^{\alpha}\left(\mathbf{x}_{J}\right)\right)$ and $\Psi_{J}^{\alpha}=\Psi\left(g^{\alpha}\left(\mathbf{x}_{J}\right)\right)$.

Unlike in XFEM, numerical integration of (18) and (19) over enriched elements does not require special attention since the strains are continuous, so techniques like in Moës et al. [2] and Ventura [29] are not needed.

When the tapered Somigliana dislocation enrichment is applied to the nodes of the element containing a dislocation core, higher-order Gauss quadrature corresponding to the order of the polynomial in (13) must be applied. Note that elements are only sub-divided for numerical integration; no additional elements are introduced. When the linear regularization is used, the superimposed element must be sub-divided along the discontinuity into two sub-elements. The number of quadrature points required for the linear regularization is much smaller than that needed for the Somigliana regularization. 


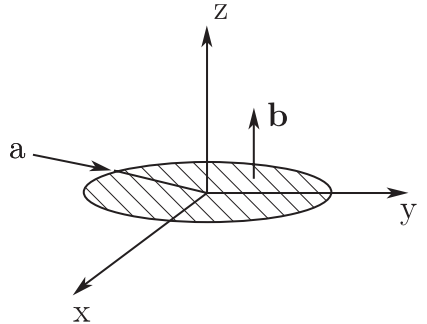

(a)

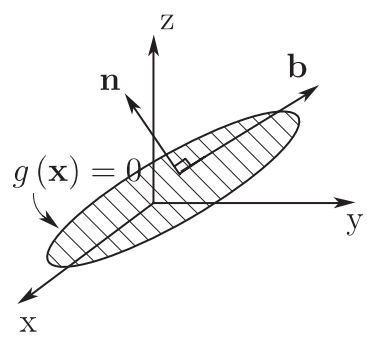

(c)

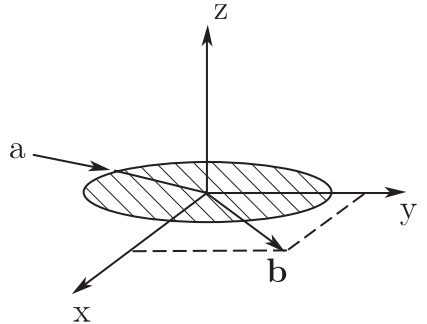

(b)

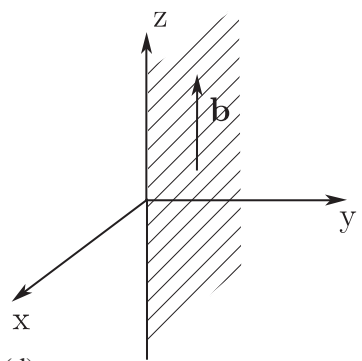

(d)

Figure 7. Illustration of several dislocations in three dimensions: (a) is a circular prismatic dislocation in the $x y$-plane; (b) is circular dislocation loop in the $x y$-plane; (c) is an arbitrary dislocation loop on an arbitrary plane; and (d) is a screw dislocation along the $z$-axis.

All of the examples to be presented use three node triangular elements. The implementation of quadrilaterals and higher-order elements is straightforward and does not introduce any difficulties in XFEM, see Stazi et al. [30].

The algorithm to determine which nodes to enrich is $\mathbf{O}\left(n^{e} \log n^{e}\right)$ per dislocation, where $n^{e}$ is the number of elements. When quasi-static simulations are performed, global searches are not required to update the enriched nodes; only local searches near the dislocation cores are required. Based on the authors' experience with XFEM for crack propagation, such local searches do not significantly increase the cost of quasi-static computations.

\subsection{Other dislocations}

We briefly give the enrichment fields for other dislocations in three dimensions, without the core regularization. The dislocations that will be considered are illustrated in Figure 7. For a prismatic dislocation loop of radius $a$ centred at the origin in the $x y$-plane, Figure $7(a)$, the enrichment is

$$
\mathbf{u}^{D}(\mathbf{x})=b_{z} \vec{k} \sum_{J \in \mathscr{P}^{\alpha}} N_{J}(\mathbf{x}) H(z) \bar{H}\left(a^{2}-x^{2}-y^{2}\right)
$$

where $\bar{H}(\cdot)=H(\cdot)+1 / 2$. For a circular mixed dislocation loop of radius $a$ centred in the $x y$-plane, Figure 7(b), the enrichment is

$$
\mathbf{u}^{D}(\mathbf{x})=\left(b_{x} \vec{i}+b_{y} \vec{j}\right) \sum_{J \in \mathscr{S}^{\alpha}} N_{J}(\mathbf{x}) H(z) \bar{H}\left(a^{2}-x^{2}-y^{2}\right)
$$


For an arbitrary mixed dislocation loop on an arbitrary plane with normal $\mathbf{n}$ and with a core defined by $g(\mathbf{x})=0$, Figure $7(\mathrm{c})$, the enrichment is

$$
\mathbf{u}^{D}(\mathbf{x})=\vec{b} \sum_{J \in \mathscr{S}^{\alpha}} N_{J}(\mathbf{x}) H\left(n_{x} x+n_{y} y+n_{z} z-c\right) \bar{H}(g(\mathbf{x}))
$$

where $c$ is a constant. For a screw dislocation in the $x z$-plane, Figure 7(d), the enrichment is

$$
\mathbf{u}^{D}(\mathbf{x})=b \vec{k} \sum_{J \in \mathscr{S}^{\alpha}} N_{J}(\mathbf{x}) H(y) \bar{H}(-x)
$$

\section{EXAMPLES}

\subsection{Dislocation in an infinite domain}

To simulate a dislocation in an infinite domain we will consider a $10^{-4} \times 10^{-4} \mathrm{~cm}$ domain containing a dislocation core with a horizontal glide plane, see Figure 8. Along the boundary ABCD we apply displacement boundary conditions corresponding to the exact solution for the infinite domain. The elastic modulus, Poisson's ratio and the magnitude of the Burgers vector are $1.2141 \times 10^{11} \mathrm{~Pa}$, 0.34 and $8.551 \times 10^{-8} \mathrm{~cm}$, respectively. An unstructured triangular mesh with about 3600 elements is used. This corresponds to an element edge length of about $100\|\mathbf{b}\|$. The analytical solution for an edge dislocation in an infinite domain, as given by Hirth and Lothe [31], is

$$
\begin{aligned}
& u_{x}=\frac{b}{2 \pi}\left[\arctan \frac{y}{x}+\frac{x y}{2(1-v)\left(x^{2}+y^{2}\right)}\right] \\
& u_{y}=-\frac{b}{2 \pi}\left[\frac{1-2 v}{4(1-v)} \ln \left(x^{2}+y^{2}\right)+\frac{x^{2}-y^{2}}{4(1-v)\left(x^{2}+y^{2}\right)}\right]
\end{aligned}
$$
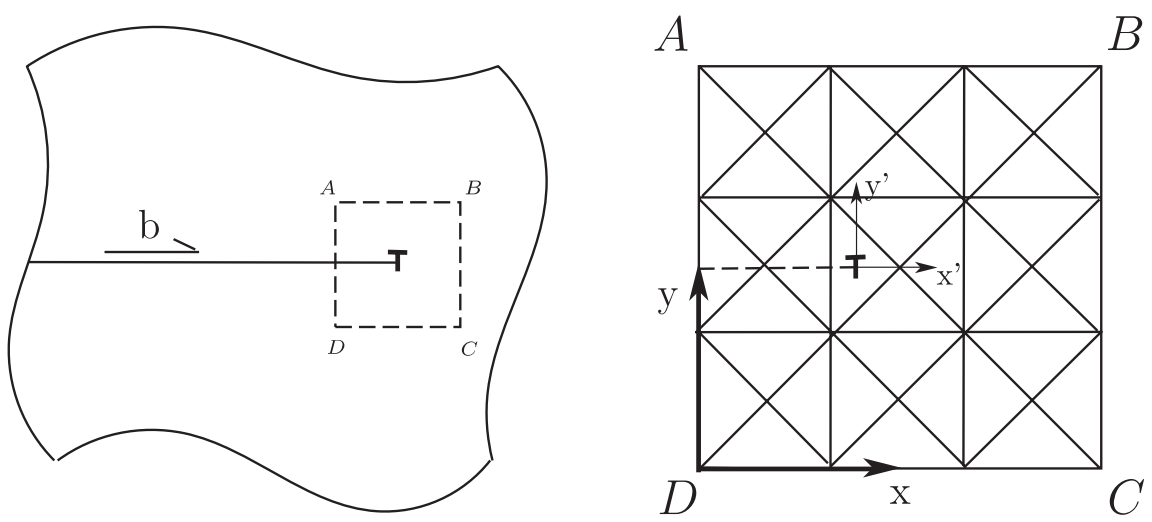

Figure 8. Illustration of the sub-domain $\mathrm{ABCD}$ of an infinite body used to simulate dislocations in an infinite domain. 

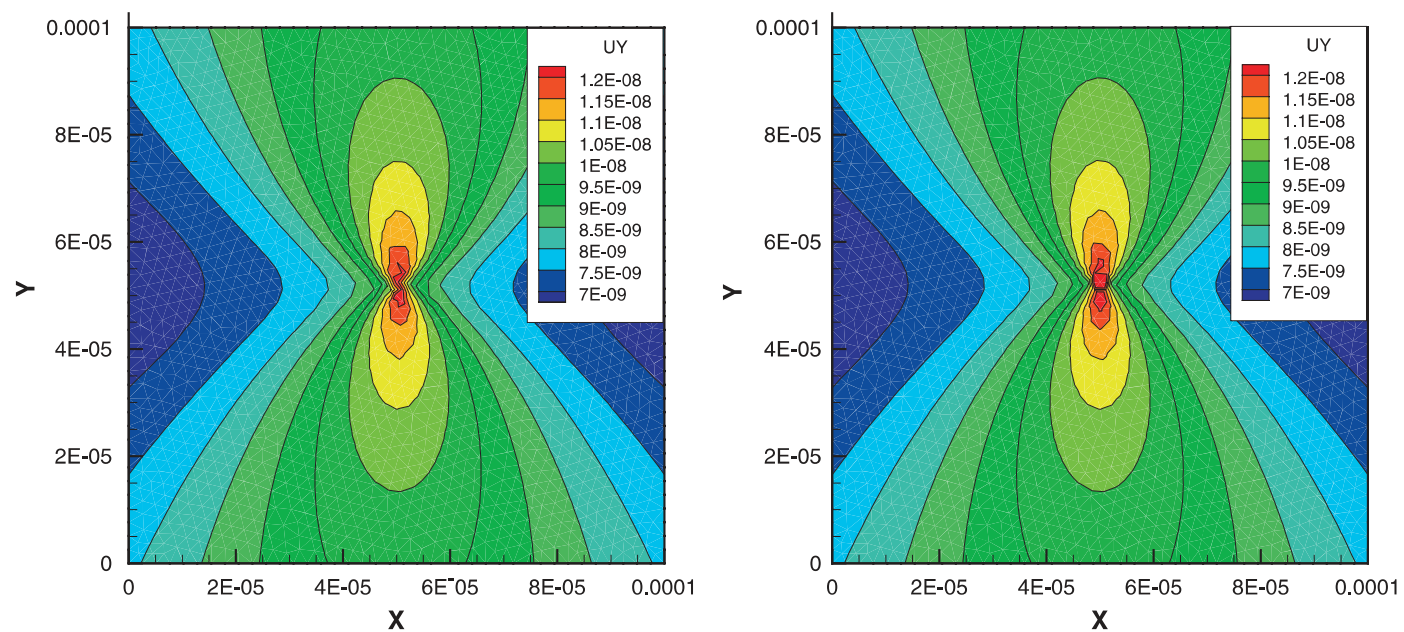

Figure 9. Displacement in the $y$-direction in $\mathrm{cm}$. On the right is the exact field; on the left are the results from XFEM.
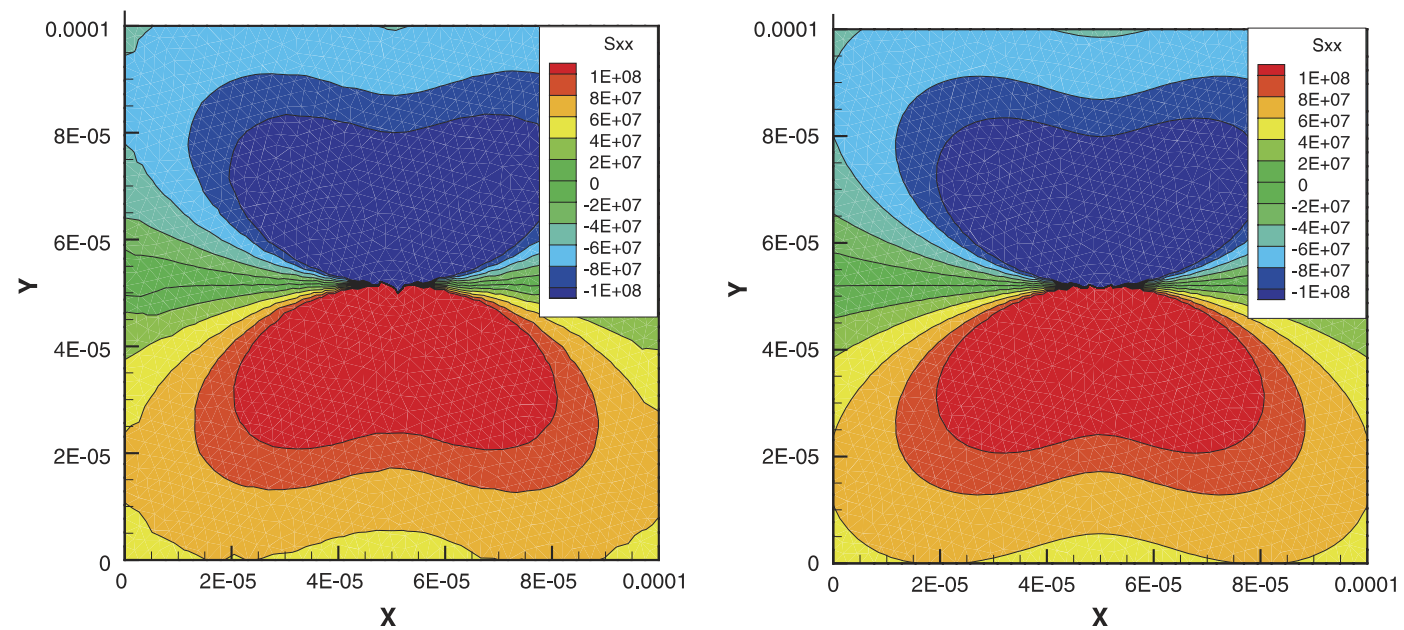

Figure 10. Stress $\sigma_{x x}$ in $10^{-1} \mathrm{~Pa}$. On the right is the exact field; on the left are the results from XFEM.

Figures 9 and 10 show that the XFEM with tangential enrichment correctly predicts the $y$-displacement and $x$-stress fields for a dislocation in an infinite domain. Similarly agreeable results were obtained for the other components of the displacement and stress.

The shear stress along the line defining the glide plane, $f(\mathbf{x})=0$, is plotted in Figure 11 with the exact solution. We see the shear stresses are accurately captured away from the dislocation core. The stress singularity is only approximately captured because the tangential enrichment approximation (15) is a regularization of the step discontinuity of the exact solution. With further mesh refinement, the regularization of the step discontinuity converges to a step discontinuity and the shear stresses near the dislocation core will increase. 


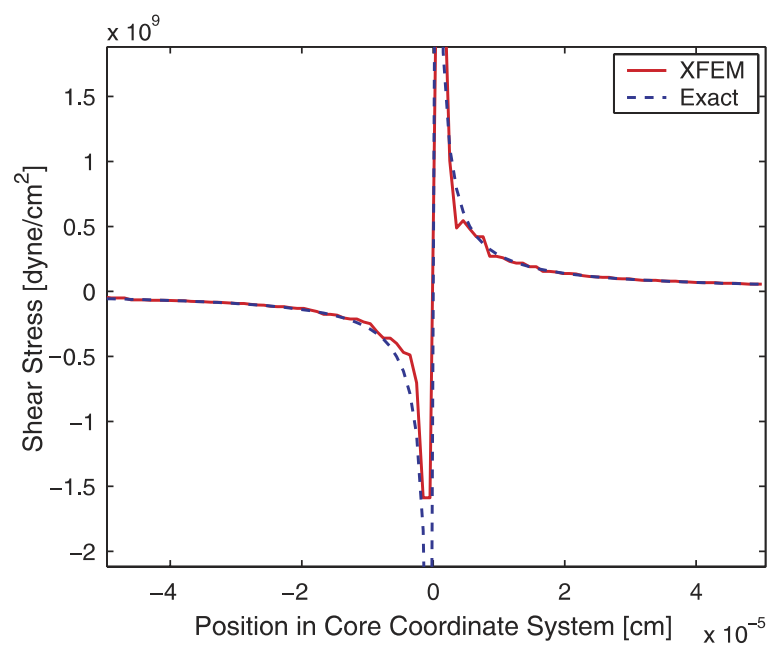

Figure 11. XFEM and exact shear stress $\sigma_{x y}$ along the glide plane of an edge dislocation in an infinite domain. The dislocation core is located at $\bar{x}=0$.

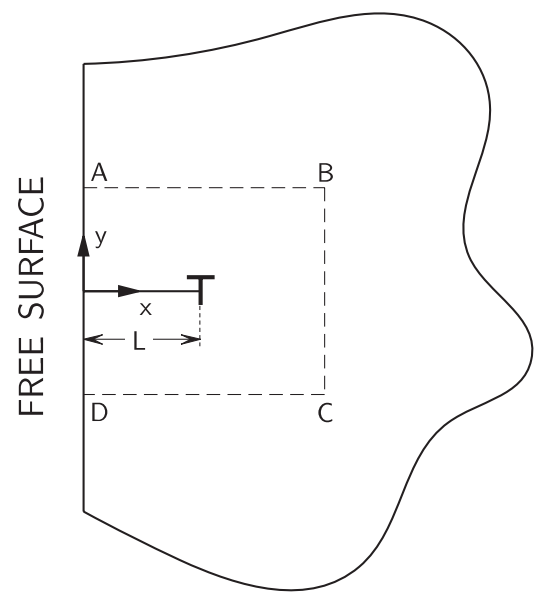

Figure 12. Edge dislocation in a semi-infinite domain, near a free surface. Sub-domain ABCD is the numerical simulation domain.

\subsection{Dislocation in a semi-infinite domain}

We consider an edge dislocation in a semi-infinite domain near a free surface, as shown in Figure 12. The free surface is located at $x=0$ and the domain is assumed to be semi-infinite and to occupy the domain $x>0$. The dislocation is located a distance of $L=0.5 \times 10^{-4} \mathrm{~cm}$ from the free surface and it is assumed that the glide plane is perpendicular to the free surface, along $y=0$. The analytical solution to this problem as given by Head [32], (after a small typographical 

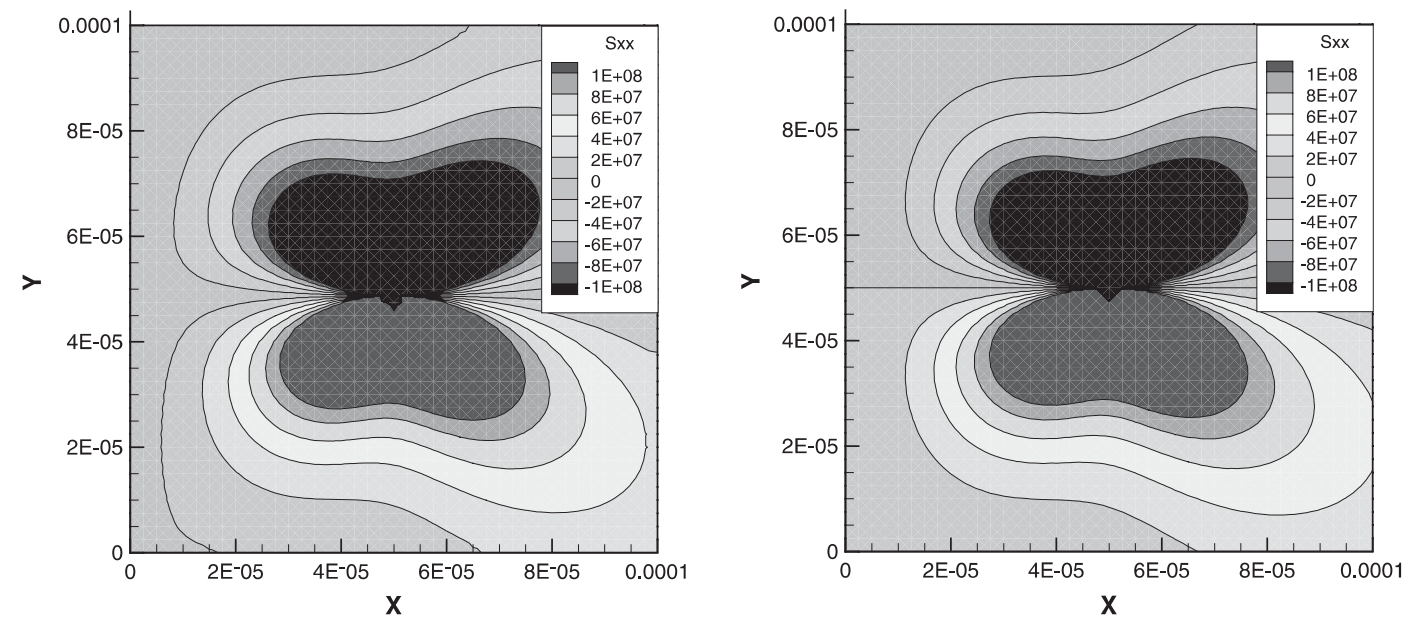

Figure 13. Stress $\sigma_{x x}$ in dyn $/ \mathrm{cm}^{2}$ for a dislocation in a semi-infinite domain. On the right is the exact field; on the left are the results from XFEM.

correction) is

$$
\begin{aligned}
\sigma_{x x}= & D\left\{-\frac{y\left\{3(x-L)^{2}+y^{2}\right\}}{\left((x-L)^{2}+y^{2}\right)^{2}}+\frac{y\left\{3(x+L)^{2}+y^{2}\right\}}{\left((x+L)^{2}+y^{2}\right)^{2}}+4 L x y \frac{\left\{3(x+L)^{2}-y^{2}\right\}}{\left((x+L)^{2}+y^{2}\right)^{3}}\right\} \\
\sigma_{y y}= & D\left\{\frac{y\left\{(x-L)^{2}-y^{2}\right\}}{\left((x-L)^{2}+y^{2}\right)^{2}}-\frac{y\left\{(x+L)^{2}-y^{2}\right\}}{\left((x+L)^{2}+y^{2}\right)^{2}}\right. \\
& \left.+4 L y \frac{\left\{(2 L-x)(x+L)^{2}+(3 x+3 L) y^{2}\right\}}{\left((x+L)^{2}+y^{2}\right)^{3}}\right\} \\
\sigma_{x y}= & D\left\{\frac{(x-L)\left\{(x-L)^{2}-y^{2}\right\}}{\left((x-L)^{2}+y^{2}\right)^{2}}-\frac{(x+L)\left\{(x+L)^{2}-y^{2}\right\}}{\left((x+L)^{2}+y^{2}\right)^{2}}\right. \\
+ & \left.2 L \frac{(L-x)(x+L)^{3}+6 x(x+L) y^{3}-y^{4}}{\left((x+L)^{2}+y^{2}\right)^{3}}\right\}
\end{aligned}
$$

where $D=E b / 4 \pi\left(1-v^{2}\right)$. We solve the problem on a sub-domain ABCD, as in Figure 12, with dimensions $10^{-4} \times 10^{-4} \mathrm{~cm}$. The sub-domain ABCD is discretized with a $40 \times 40$ cross-triangular element mesh, like the one shown in Figure 8. Traction boundary conditions corresponding to the analytical solution (31) are applied on all boundaries other than the free surface.

The stress fields $\sigma_{x x}$ and $\sigma_{x y}$ are compared to the exact solution (31), in Figures 13 and 14, respectively. From this example it is clear that the method can accurately capture the dislocation fields. The relative energy error norm is

$$
\text { Relative energy error norm }=\frac{W\left(\boldsymbol{\varepsilon}-\boldsymbol{\varepsilon}^{h}\right)}{W(\boldsymbol{\varepsilon})}
$$



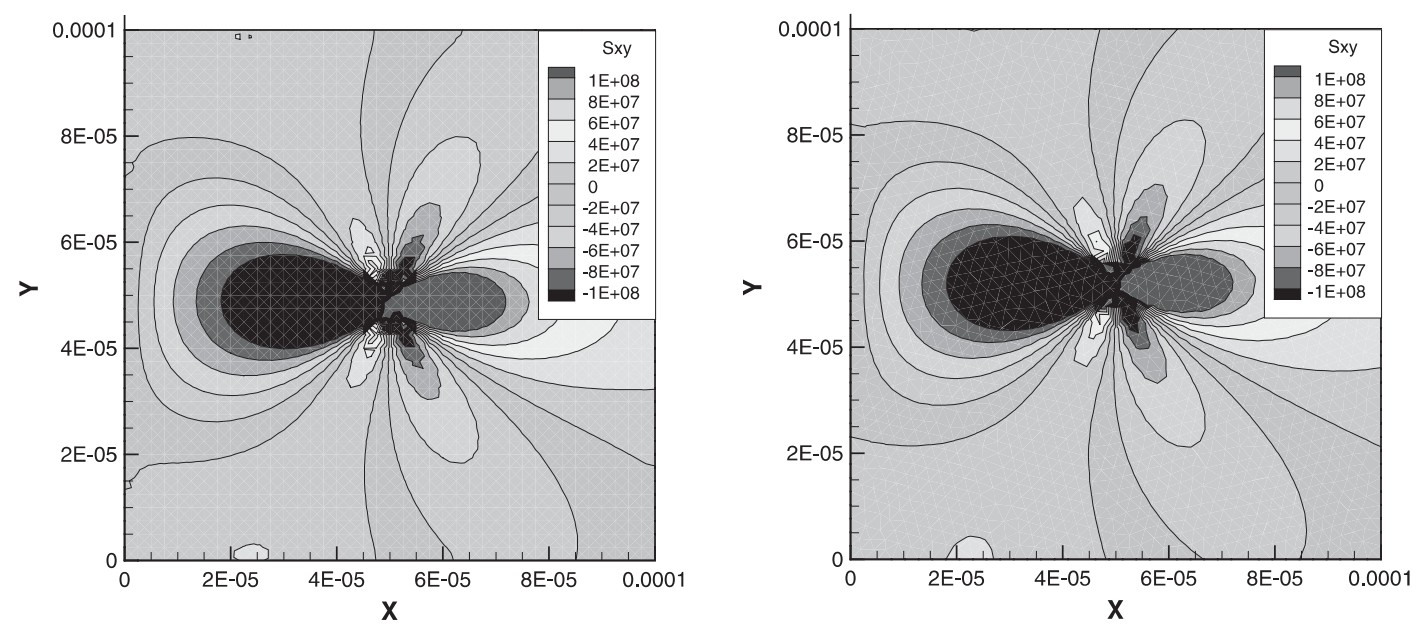

Figure 14. Shear stress $\sigma_{x y}$ in dyn $/ \mathrm{cm}^{2}$ for a dislocation in a semi-infinite domain. On the right is the exact field; on the left are the results from XFEM.

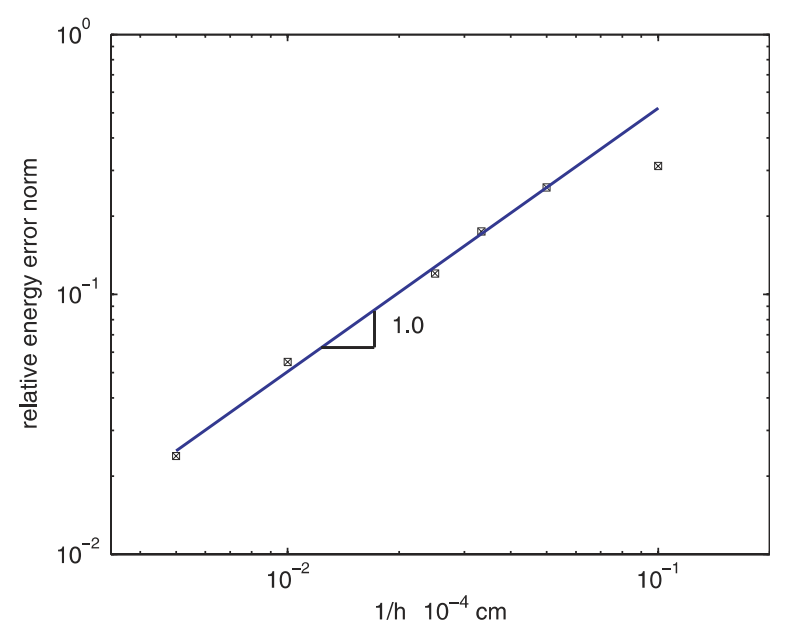

Figure 15. Convergence of the relative energy error norm with decreasing element size, $h_{e}$. The convergence rate is 1.0 .

where

$$
W(\varepsilon)=\left[\int_{\Omega / \Gamma_{d}} \boldsymbol{\varepsilon}: \mathbf{C}: \boldsymbol{\varepsilon} d \mathbf{\Omega}\right]^{1 / 2}
$$

and $\boldsymbol{\varepsilon}$ is the analytical solution, $\varepsilon^{h}$ is the numerical solution and $\mathbf{C}$ is the elasticity tensor. The convergence of the relative energy error norm is shown in Figure 15. Since the strain energy in the vicinity of the core diverges with mesh refinement, the energy within a distance of $0.05 \mu \mathrm{m}$ 


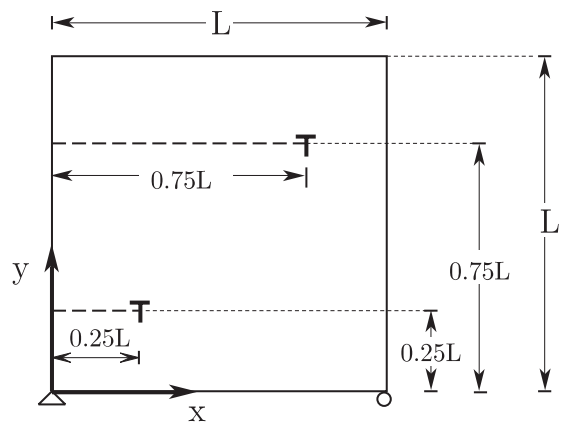

Figure 16. Two dislocations in a square, $L \times L$, simply supported domain with traction free boundaries.

Dashed line represents the dislocation glide planes.
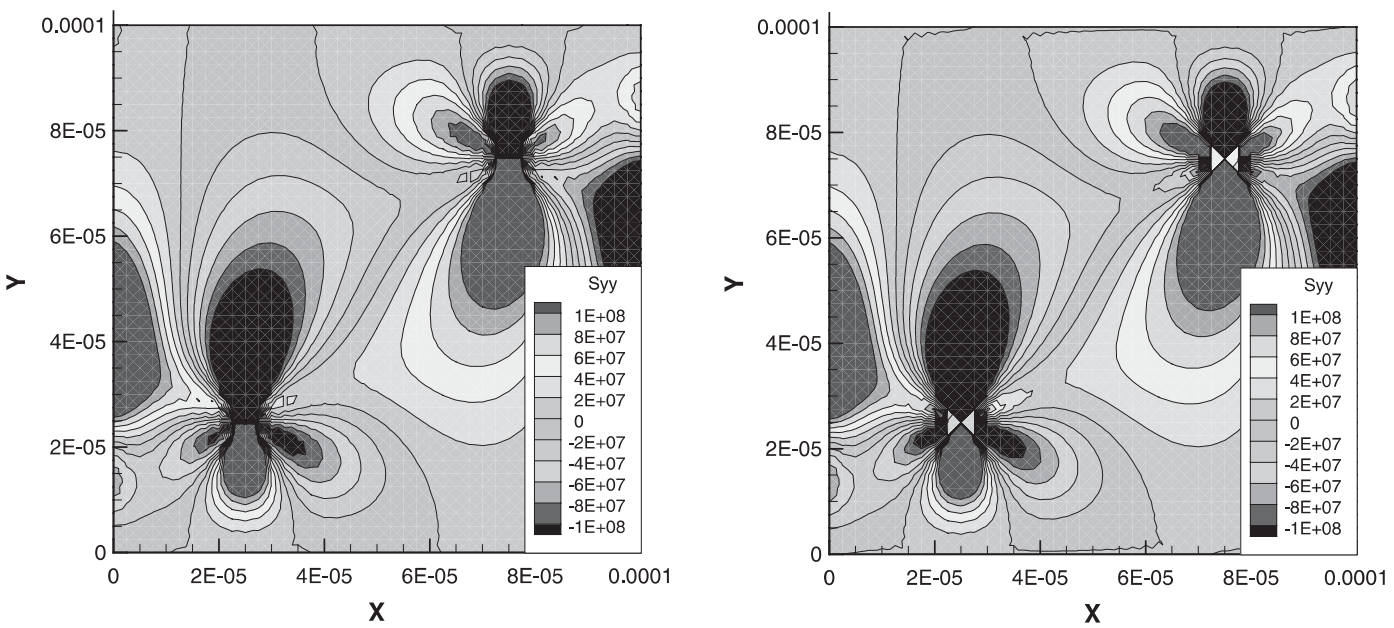

Figure 17. Stress $\sigma_{y y}$ in $10^{-1} \mathrm{~Pa}$ for two dislocations in a domain with traction-free boundary conditions. On the left are the results from XFEM; on the right are the results from the method of van der Giessen and Needleman [4].

from the core was neglected in the integration of (33). The convergence rate of the method is 1.0 which is the optimum rate for linear finite elements.

\subsection{Dislocations in a plate without boundary tractions}

The next example illustrates the ability of XFEM to capture dislocation interactions. The results are compared with those from the method of van der Giessen and Needleman [4]. We consider a square domain with dimensions $L \times L$ containing two edge dislocations, as shown in Figure 16 . The boundaries are traction-free; rigid body motion is precluded by constraining three degrees of freedom. The glide planes are parallel to the $x$-axis and the cores are located at $(0.75 \mathrm{~L}, 0.75 \mathrm{~L})$ and $(0.25 L, 0.25 L)$. We will consider a square domain with sides of length $L=10^{-4} \mathrm{~cm}$ with elastic modulus $1.2141 \times 10^{11} \mathrm{~Pa}$, Poisson's ratio 0.34 , and the Burgers vector of the two dislocations $\|\mathbf{b}\|=8.551 \times 10^{-8} \mathrm{~cm}$. 


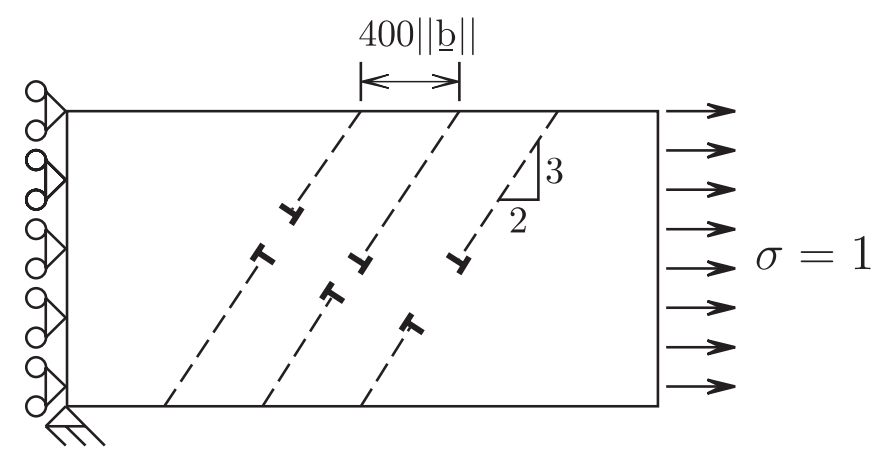

Figure 18. Rectangular domain under uniaxial tension. Dashed lines represent glide planes.
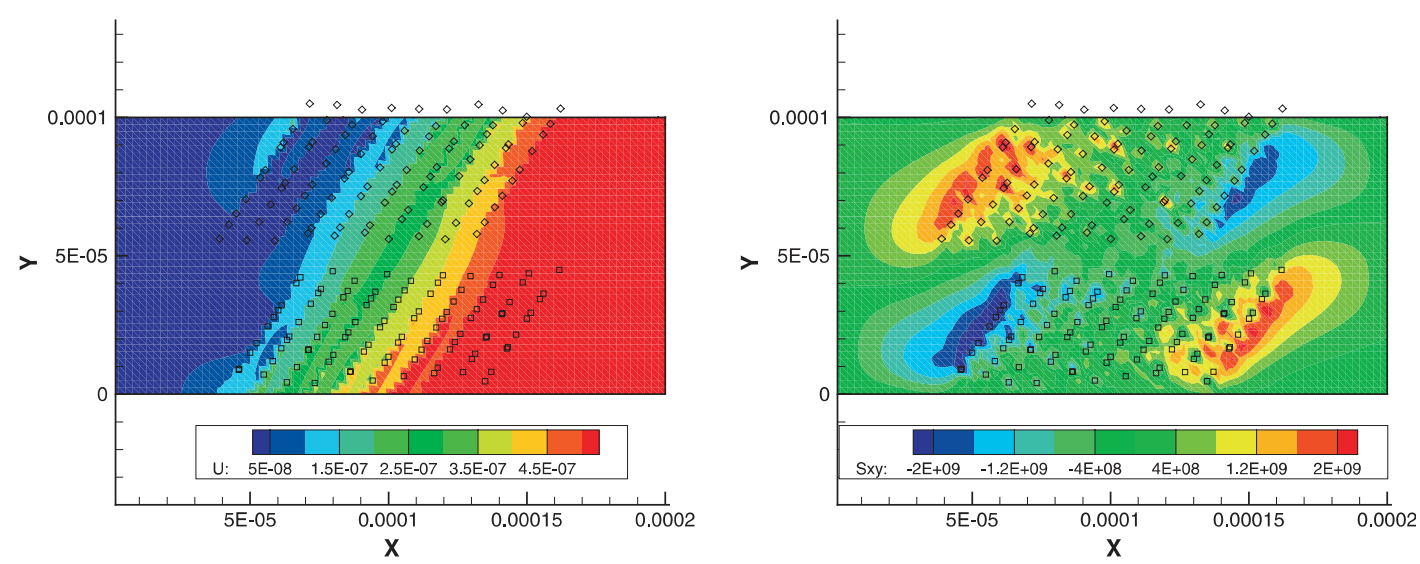

Figure 19. Contour plots of a uniaxial tension specimen with 200 dislocations. Left is the displacement field; right is the shear stress $\sigma_{x y}$ contours. Squares represent dislocation cores with positive Burgers vectors and diamonds represent dislocations with negative Burgers vectors.

The stress $\sigma_{y y}$ contours from XFEM and the method of van der Giessen and Needleman [4], for a $40 \times 40$ cross-triangle element mesh are shown in Figure 17. As can be seen from this figure, XFEM compares well with [4]. Other displacement and stress fields agree equally well.

\subsection{Plate under uniaxial loading}

We next illustrate the use of the proposed method for problems with a large number of dislocations. We will consider a rectangular domain with dimensions $2 \times 10^{-4} \times 10^{-4} \mathrm{~cm}$, elastic modulus $1.2141 \times 10^{12} \mathrm{dyn} / \mathrm{cm}^{2}$ and Poisson's ratio 0.34. A system of 200 edge dislocations with Burgers vectors $\left\|\mathbf{b}^{\alpha}\right\|=8.551 \times 10^{-8} \mathrm{~cm}, \alpha=1,2, \ldots, 200$, are considered on 14 parallel slip planes spaced $400\|\mathbf{b}\|$ apart with a slope of $\frac{3}{2}$, see Figure 18 . A tensile load of $1 \mathrm{dyn} / \mathrm{cm}^{2}$ is applied to the right boundary. The left edge of the domain is fixed in the $x$-direction. Rigid body motion is constrained by fixing the node in the bottom left corner of the domain. The displacement and shear stress $\sigma_{x y}$ contours for a uniform $80 \times 40$ element mesh are shown in Figure 19. To give a qualitative 
measure of the efficiency of the proposed method, the execution time for the assembly and solution of the Matlab code on a single CPU PC was about $20 \mathrm{~s}$.

\section{CONCLUSIONS}

In the proposed method for modelling dislocations, slip is prescribed on internal surfaces. The solution of dislocation fields can then be simply viewed as the solution of elasticity equations (3)-(5) subject to internal displacement boundary conditions. This is consistent with the concepts of Volterra [25] and Eshelby [26], where the stress due to a dislocation is completely characterized by the specification of the slip across the glide plane.

The accuracy of the proposed method and its effectiveness for the simulation of large numbers of dislocations has been demonstrated. The proposed method does not depend on superposition; instead slip is prescribed explicitly on internal surfaces and the boundary value problem is solved using an XFEM approximation with tangential enrichment. Since the slip across the glide plane is represented explicitly and exactly, the proposed method accurately captures dislocation fields. Accurate finite energy dislocation cores such as the tapered Somigliana dislocation enrichment can be incorporated into the model by core enrichment functions. These provide a natural regularization of the dislocation energy, which is an important feature of this method. Though not considered here, other core enrichment functions can be constructed in accordance with experimental or atomistic simulation results. The presentation here has been limited to edge dislocations; however, the extension of the method to other types of dislocations is straightforward.

\section{ACKNOWLEDGEMENTS}

This work was supported by the Office of Naval Research under Grant N00014-03-1-1097, the Army Research Office under Grant W911NF-05-1-0049 and by a Graduate Scholarship from the Natural Sciences and Engineering Research Council of Canada.

\section{REFERENCES}

1. Belytschko T, Black T. Elastic crack growth in finite elements with minimal remeshing. International Journal for Numerical Methods in Engineering 1999; 45:601-620.

2. Moës N, Dolbow J, Belytschko T. A finite element method for crack growth without remeshing. International Journal for Numerical Methods in Engineering 1999; 46:131-150.

3. Belytschko T, Moës N, Usui S, Parimi C. Arbitrary discontinuities in finite elements. International Journal for Numerical Methods in Engineering 2001; 50:993-1013.

4. van der Giessen E, Needleman A. Discrete dislocation plasticity: a simple planar model. Modelling Simulation in Material Science and Engineering 1995; 3:689-735.

5. Amodeo RJ, Ghoniem NM. Dislocation dynamics. I. A proposed methodology for deformation micromechanics. Physical Review B 1990; 41:6958-6967.

6. Schwarz KW, Tersoff J. Interaction of threading and misfit dislocations in a strained epitaxial layer. Applied Physics Letters 1996; 69:1220-1222.

7. Canova G, Brechet Y, Kubin LP, DeVincre B, Pontikis V, Condat M. 3d Simulation of dislocation motion on a lattice: application to the yield surface of single crystals. Solid State Phenomena 1993; 35-36:101-106.

8. Zbib HM, Rhee M, Hirth JP. On plastic deformation and the dynamics of $3 \mathrm{~d}$ dislocations. International Journal of Mechanical Sciences 1998; 40:113-127.

9. Devincre B, Kubin LP, Lemarchand C, Madec R. Mesoscopic simulations of plastic deformation. Material Science and Engineering 2001; A309-310:211-219. 
10. Lemarchand C, Devincre B, Kubin LP. Homogenization method for a discrete-continuum simulation of dislocation dynamics. Journal of the Mechanics and Physics of Solids 2001; 49(9):1969-1982.

11. Sukumar N, Moës N, Moran B, Belytschko T. Extended finite element method for three-dimensional crack modelling. International Journal for Numerical Methods in Engineering 2000; 48:1549-1570.

12. Moës N, Belytschko T. Extended finite element method for cohesive crack growth. Engineering Fracture Mechanics 2002; 69:813-833.

13. Song J-H, Areias PMA, Belytschko T. A method for dynamic crack and shear band propagation with phantom nodes. International Journal for Numerical Methods in Engineering 2006; 67(6):868-893.

14. Sukumar N, Chopp DL, Moës N, Belytschko T. Modeling holes and inclusions by level sets in the extended finite-element method. Computer Methods in Applied Mechanics and Engineering 2001; 190:6183-6200.

15. Chessa J, Belytschko T. An extended finite element method for two-phase fluids. Journal of Applied MechanicsTransactions of the ASME 2003; 70(1):10-17.

16. Xiao QZ, Karihaloo BL. Improving the accuracy of xfem crack tip fields using higher order quadrature and statically admissible stress recovery. International Journal for Numerical Methods in Engineering 2006; 66(9): 1378-1410.

17. Legay A, Wang HW, Belytschko T. Strong and weak arbitrary discontinuities in spectral finite elements. International Journal for Numerical Methods in Engineering 2005; 64(8):991-1008.

18. Laborde P, Pommier J, Renard Y, Salaun M. High-order extended finite element method for cracked domains. International Journal for Numerical Methods in Engineering 2005; 64(3):354-381.

19. Rethore J, Gravouil A, Combescure A. A combined space-time extended finite element method. International Journal for Numerical Methods in Engineering 2005; 64(2):260-284.

20. Areias PMA, Belytschko T. Analysis of three-dimensional crack initiation and propagation using the extended finite element method. International Journal for Numerical Methods in Engineering 2005; 63(5):760-788.

21. Legrain G, Moës N, Verron E. Stress analysis around crack tips in finite strain problems using the extended finite element method. International Journal for Numerical Methods in Engineering 2005; 63(2):290-314.

22. Larsson R, Fagerstrom M. A framework for fracture modelling based on the material forces concept with xfem kinematics. International Journal for Numerical Methods in Engineering 2005; 62(13):1763-1788.

23. Areias PMA, Belytschko T. Two-scale shear band evolution by local partition of unity. International Journal for Numerical Methods in Engineering 2006; 66(5):878-910.

24. Samaniego E, Belytschko T. A continuum-discontinuum modelling of shear bands. International Journal for Numerical Methods in Engineering 2005; 62(13):1857-1872.

25. Volterra V. Sur l'quilibre des corps lastiques multiplement connexes. Annales Scientifiques de l'cole Normale Suprieure Sr. 3 1907; 24:401-517.

26. Eshelby JD. The force on an elastic singularity. Philosophical Transactions of the Royal Society of London. Series A, Mathematical and Physical Sciences 1951; 244(244):87-112.

27. Ventura G, Moran B, Belytschko T. Dislocations by partition of unity. International Journal for Numerical Methods in Engineering 2005; 62(11):1463-1487.

28. Melenk JM, Babuška I. The partition of unity finite element method: basic theory and applications. Computer Methods in Applied Mechanics and Engineering 1996; 139:290-314.

29. Ventura G. On the elimination of quadrature subcells for discontinuous functions in the extended finite element method. International Journal for Numerical Methods in Engineering 2006; 66(5):761-795.

30. Stazi FL, Budyn E, Chessa J, Belytschko T. An extended finite element method with higher-order elements for curved cracks. Computational Mechanics 2003; 31:38-48.

31. Hirth JP, Lothe J. Theory of Dislocations, vol. 1 (2nd edn). Wiley: New York, 1982.

32. Head AK. Edge dislocations in inhomogeneous media. Proceedings of the Physical Society, Section B 1953; 66(9):793-801. 